\title{
"PS for You": A Contactless, Remote, Cloud-Based Body Measurement Technology Powered by Artificial Intelligence
}

\author{
Francesca DONADONI ${ }^{1}$, Jayne MECHAN ${ }^{2}$, Colette JOHNSON *1 \\ ${ }^{1}$ The PS Collective, New York City, USA; \\ ${ }^{2}$ Manchester Metropolitan University, Manchester, UK \\ https://doi.org/10.15221/21.31
}

\begin{abstract}
Contactless and remote measurement technologies have recently been at the core of the digital shopping experience, not only for their potential to provide better customization but to revolutionize the way customers shop - from minimizing returns due to misfits to reducing environmental impact by producing on-demand. For small-batch retailers, this is key in ensuring minimal waste and streamlining the consumer journey. From the consumer's point of view, fit is also an unaddressed issue. After conducting user interviews on $60+$ women, we identified $70 \%$ of respondents reported difficulties finding proper fitting apparel by designers. Reports by Mintel [1] and Deloitte [2] also confirm that the issue of fit is paramount in the industry. Here we introduce "PS for you", our Al-powered solution to provide a fast and scalable platform for designers to obtain reliable measurements and for shoppers to find the right fit.
\end{abstract}

Our solution uses computer vision models paired with a proprietary layer of post-processing algorithms to extract personalized measurements from photos that the user can upload from any device. The solution is deployed on a serverless CPU using Amazon Web Services (AWS) for easier interoperability and faster run times.

To test the effectiveness of this approach, we collected 40 images from 20 volunteers of different body shapes and sizes (including petite, short-stature, and athletic). The system was able to detect measurements for waist, hips, and bust with an average difference of less than $3 \%$ with respect to tapemeasured data. The median difference remained $<1 \mathrm{in}$, a significant improvement which is $3 x$ more reflective of true size than the current universal sizing methods [3].

Working on this technology allows us to foster a new connection between shoppers interested in customization, sustainability, and comfort and designers who are interested in decreasing returns, eliminating waste, and understanding more about their consumers. Technology will not just help but can create opportunities to build new systems, without heavy manual support that connects and fosters experiences anywhere in the World. By using the right technology and data, responsibly, we're building a product that will open up opportunities in connected markets as well as enhancing existing ones.

Keywords: Machine learning and artificial intelligence for 3D body modeling and processing, Portable human body measurement systems, Apparel and fashion, Custom apparel, Digital anthropometry

\section{Introduction}

Computer vision applications are already pervasive in our daily lives: from the ability to unlock our phones with our eyes to uploading credit card information by scanning technology [4]. Recent advancements have allowed a number of applications to benefit from this technology, as seen in popular use cases across remote surveillance and robotics [5]. As a general definition, computer vision is the ensemble of mathematical and computational techniques to recover properties of the physical world, such as shape, illumination, and color distribution, from images [6]. In retail, most advancements have been focused on packaged goods with the goal of reducing inventory [7] and automating, both, shelfstocking [8] and checkouts [9]. When analyzing the more narrow sector of fashion and online shopping, potential applications of computer vision range from item retrieval and suggestion, to style learning and compatibility, to physical simulation and virtual try-ons [10].

All the above applications can be enhanced by layering on accurate sizing technology. The business value of reducing returns in the industry has been estimated at a potential savings of up to $\$ 20 \mathrm{~B}$ [11]. By developing a computer vision application focused on body measurement, brands can directly address the number one issue leading to returns...sizing.

*info@pscollective.co 
Currently, mass-produced clothing still relies on statistical studies that started in the 1940s [12], with the most recent updates in the 2010s [13]. This, in addition to vanity sizing, has contributed to the unreliability of the sizing system today [14]. As bodies in the US are changing, standard apparel sizing has become increasingly obsolete: with the indisputable rise of online shopping, this has become a major barrier to the user experience. In addition, women of "diverse" sizing cohorts (plus-size, petite, disabled, etc.), who are the majority of women in the US, are often left with a very limited selection of clothing that doesn't fit or exude their style [15]. This, in turn, can affect confidence and, ultimately, happiness as shown in multiple research studies [16], [17]. The current fashion environment forces a majority of women to feel unseen, unheard, and less confident [18], [19], while more personalized experiences, for instance through the use of avatars, have been shown to have a neutral effect [20].

In the US, custom or bespoke clothing is most commonly seen applied to wedding dresses or men's suits, as opposed to other countries where tailoring has deep roots entrenched in culture and even symbolizes prosperity or luxury [21]. Despite this, the search for opportunities to provide value for customers through customization, and using technology to manage realistic benefit-cost ratios for both the producer and the customer, has been active and proven successful for some time now [22]. With recent advancements in the field of $\mathrm{Al}$, the issue has now become that of ensuring technology can continue to support this transition to mass customization without the risk of perpetuating prejudice and biases.

To this end, using body scanning can provide a truly personalized solution that is fully tailored to the customer. Body scanning technologies are currently being used in both research and commercial settings. There is a variety of $3 D$ scanners available at a wide range of prices, as well as mobile applications such as MySizeID, 3DLook, and Me Three Sixty that can be used without the need for hardware [23]. These technologies have the potential to revolutionize the customer experience, however, they are often monolithic systems, have limited functionality, were developed with traditional sizing methods as a baseline, or come at too high a cost for small businesses to utilize. We are focusing on developing a solution that can easily be integrated into small businesses' online stores and is not dependent on traditional sizing methods: each size is fully personalized to the customer.

At The PS Collective, we are developing a modular technology using computer vision algorithms to estimate body measurements used by businesses to create bespoke clothing. Our "PS for you" system is built as an API that can be easily integrated into other platforms used frequently by our designers/brands, such as Shopify. After users submit two photos, the core function, built using computer vision models, recognizes the body shape and key points along the body, then applies postprocessing algorithms to extract 24 body measurements. In order to enable easy deployment of this technology in current fashion designers' websites, we packaged the system as a cloud API using Docker, AWS Lambda, and Gateway.

In this paper, we describe the development of the solution: the method section outlines the product ideation and development process, with a focus on the Al models and cloud infrastructure used; the following section describes the process of data collection for testing with details on the demographic of the sample used; the results section presents data on selected metrics used for this first validation of the system, and finally, we conclude with key insights and future improvements.

\section{Method}

\subsection{Background Research}

In our discovery phase, we interviewed a sample of 60 individuals to assess the current demand for body scanning technology for remote body measurement. Of the 57 individuals in the sample who identified as women, 95\% agreed that they would pay more for brands that fit and $71 \%$ are interested in sizing tools to help ensure a proper fit.

\subsection{Al Algorithms}

Given interest in such a system to aid in the online shopping experience, we focused on finding existing open-source algorithms that could be adapted and expanded for this purpose. The algorithms that output key points and contours in our application are available on Tensorflow and PyTorch. To adapt these technologies for our purpose, we developed a layer of post-processing functions working in collaboration with designers using OpenCV and Python. This work resulted in an Al application able to detect, from two input images, a range of reference measurements used in traditional tailoring and 
bespoke design, outlined in Figure 1. This selection of measurements was shortlisted after interviewing 30 sustainable designers who shared with us the measures they would normally take if preparing custom pieces. For the purpose of this study, we are focused on the traditional bust-waist-hips measurements for validation of the technique.

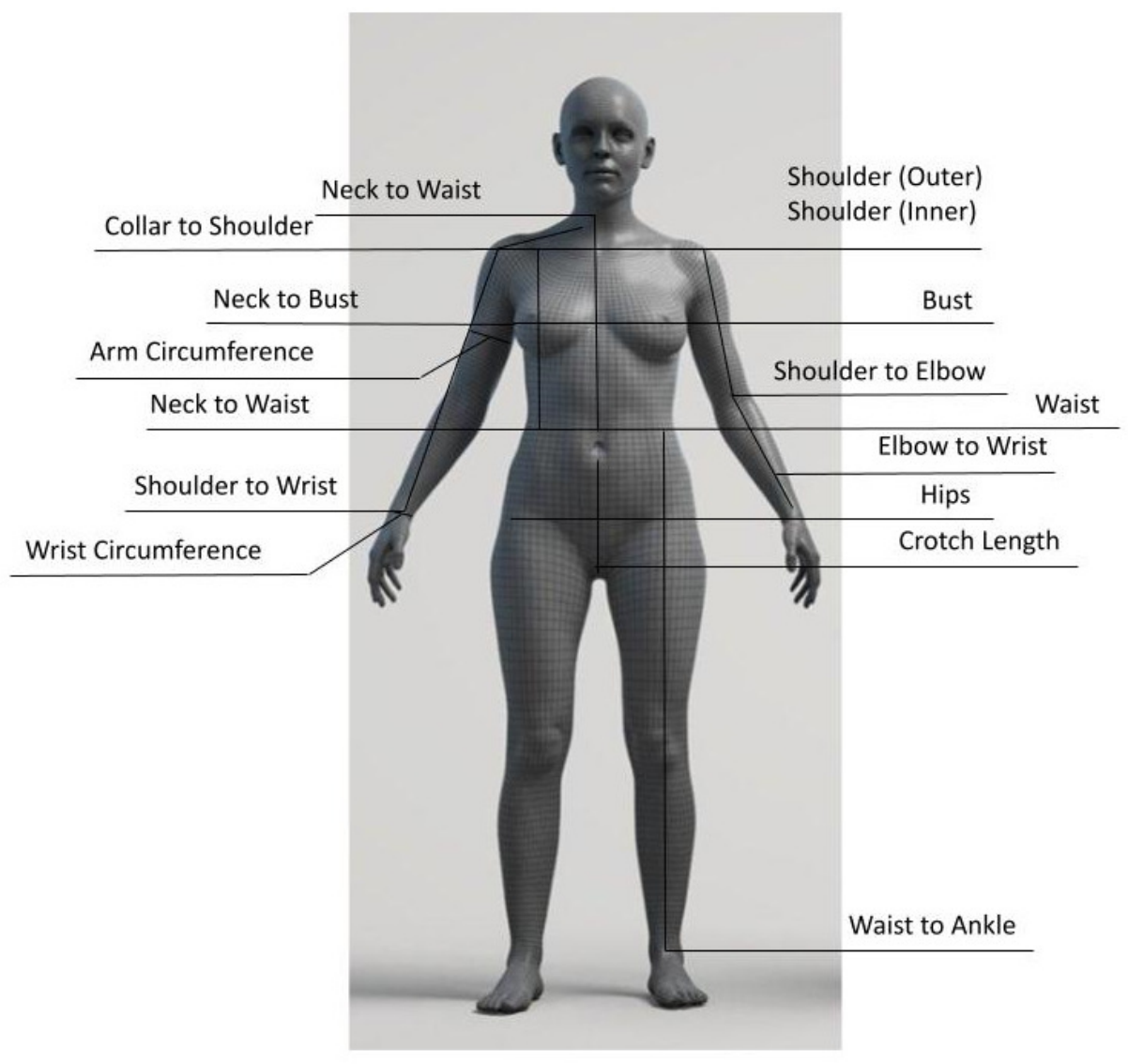

Figure 1: Set of measurements detected by the PS for You system using image segmentation, keypoint detection, and a proprietary post-processing set of algorithms.

Currently, most size recommendation technologies use big data to infer customers' size based on historical purchases made by similar users [24]. The difficulty in this approach is that body shapes present high variability, even when many data points are equal: customers of the same height, weight, and age could have very different body shapes, other-abilities, preferences, and other factors that contribute to wearing different sizes. Despite minute shifts in improved user experience, the difficulty in generalizing audiences causes an even larger limitation [25]. Our approach targets full personalization, made possible by the latest enhancements in mobile digital cameras and the development of accurate computer vision techniques. Instead of inferring size based on data generated by the same flawed, standardized system, we are using novel techniques that allow estimating the size based on actual shoppers' data.

\subsection{Cloud System}

The next step in the development of our solution was to ensure easy deployment and integration into existing applications. In order to achieve good performance under these aspects, we developed a cloud API. Using Amazon Web Services (AWS), we were also able to design a serverless solution that provides a more cost-effective alternative to a setup that uses a computing instance running on the cloud continuously. The system is a single-purpose application that leverages Lambda, Gateway, and S3. Machine learning templates available within AWS SAM accelerated our development process. The Al capabilities are achieved through the use of Python and relevant libraries (OpenCV, PyTorch, and Tensorflow), all containerized in a Docker image. Despite having a heavy code base (450 MB), we were able to deploy on Lambda using containerization with Docker. The full data pipeline is summarized in Figure 2. 


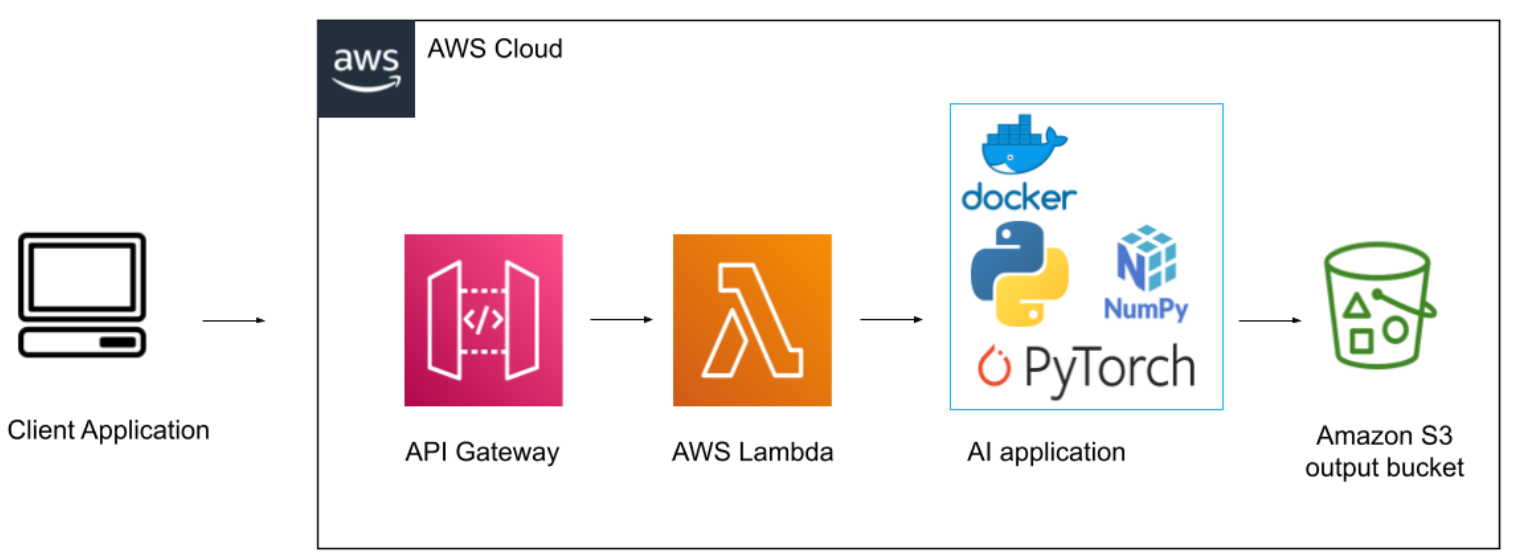

Figure 2: Using AWS cloud services we implemented a cost-effective and robust system to deploy the computer vision models that infer body measurements from customers' images.

The API takes the following inputs: URL links to two full-body images (front and sideways) of the customer, height and bra size. Through AWS Gateway, the API calls the models that are stored on AWS Lambda and run on serverless CPU, taking approximately 7 minutes to complete. The results are saved on AWS S3 and can be accessed from any application.

AWS Lambda provides a cost-effective, scalable, and robust system to deploy computer vision models that can run on CPU for inference [26].

\section{Data}

In order to test our system, we collected full body front and sideways input images, along with height, bra size, and bust-waist-hips measurements for 20 volunteers who self-identified as female and gave informed consent to the use of their anonymized data (a total of 40 images was collected with corresponding tabular data). A summary of the data collected for each volunteer is shown in Figure 3.

\begin{tabular}{|c|}
\hline Height (ft in) \\
\hline Bra size \\
\hline Bust (in) \\
\hline Waist (in) \\
\hline Hips (in) \\
\hline
\end{tabular}

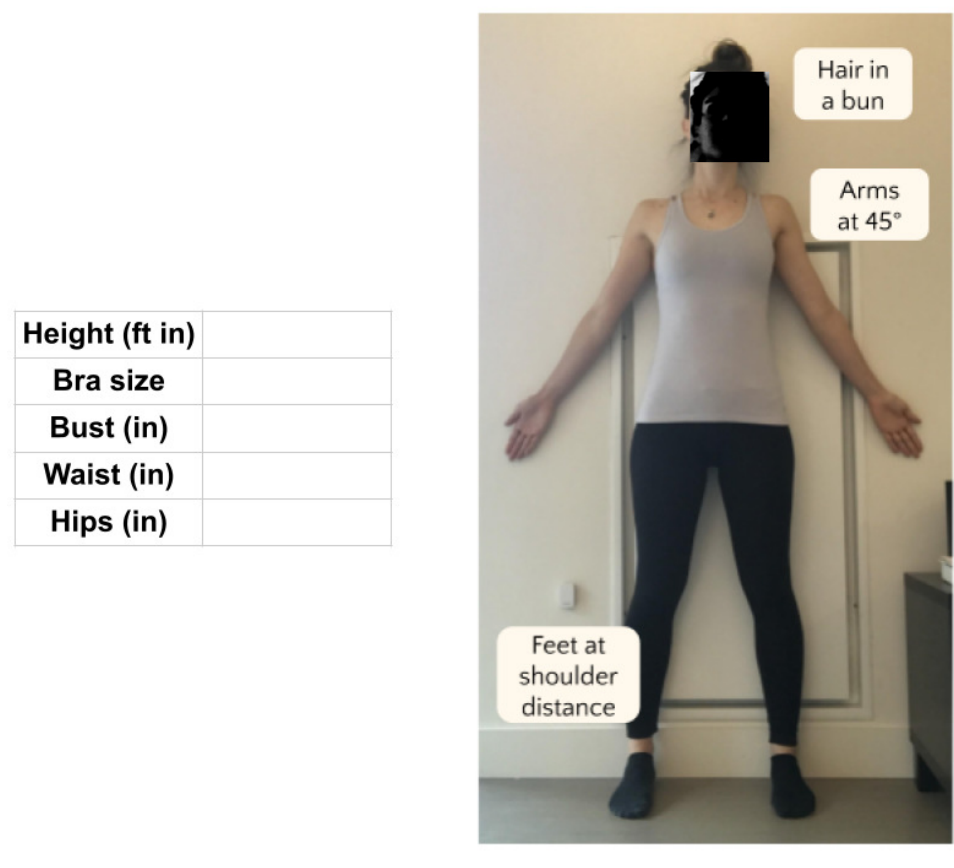

Figure 3: Example of the data collected. Only the two images, height and bra size are requested to the customer, while the bust-waist-hips measurements are used for validation.

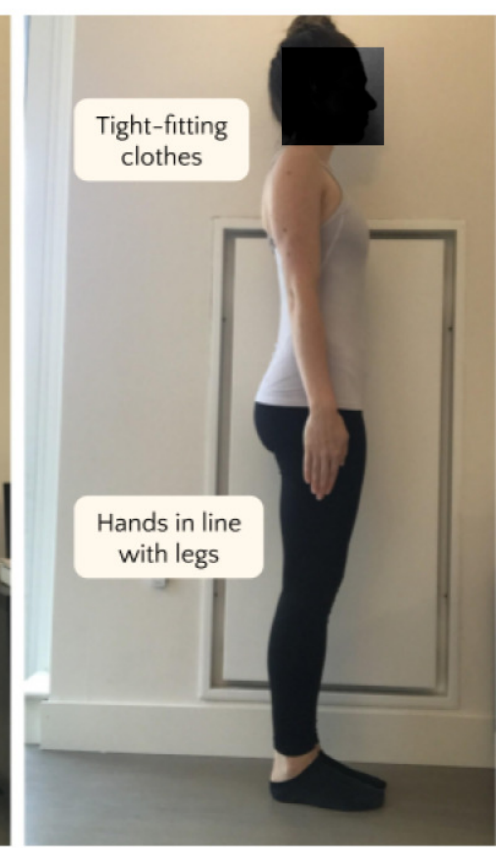


Eighty percent of the data (16 data points) was collected through the platform prolific.co [27], while the remaining was gathered in person. Prolific is a platform used for research that lets researchers select the profile sought for a certain survey or data collection, and reaches out to thousands of contributors online. The average height was $164.85 \mathrm{~cm}$, with a minimum $150 \mathrm{~cm}(4 \mathrm{ft} 11 \mathrm{in})$ and a maximum of $178 \mathrm{~cm}$ (5ft 10in). In comparison, the average height of an American woman is a few centimeters shorter than in the sample used for this study, at $161 \mathrm{~cm}$ (63.5in) [28].

As shown in Figure 4, the height of the participants was skewed towards the petite size, while the bust, waist, and hips measurements generally gravitate around what would be considered a size Small.

a)

Distribution of participants' height (ft)

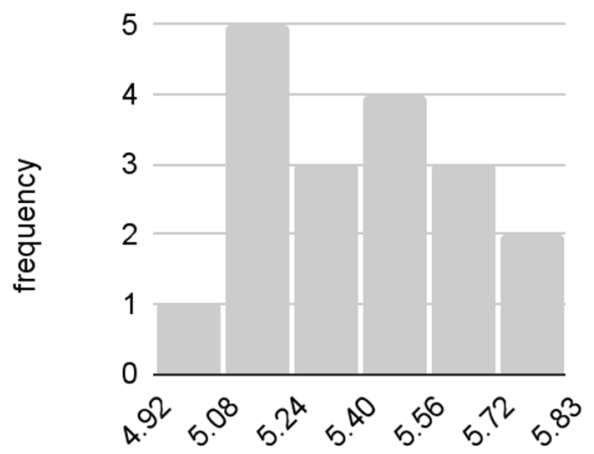

c)

Distribution of waist measurements (in)

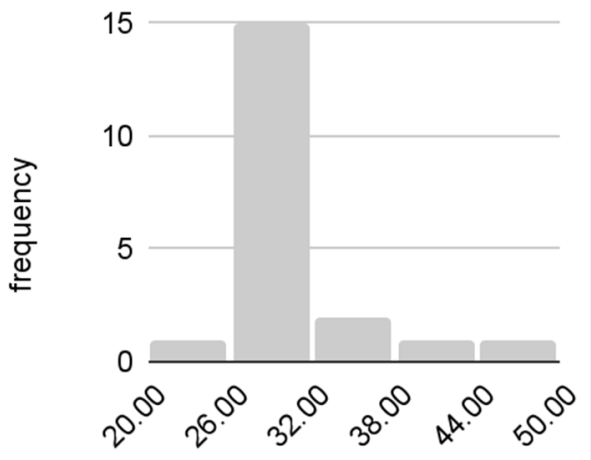

b)

Distribution of bust measurements (in)

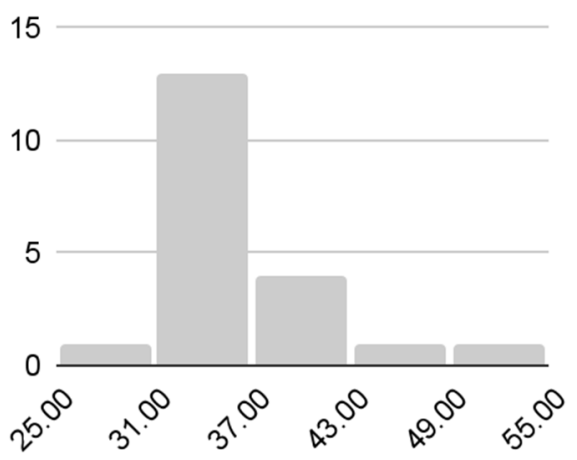

d)

Distribution of hips measurements (in)

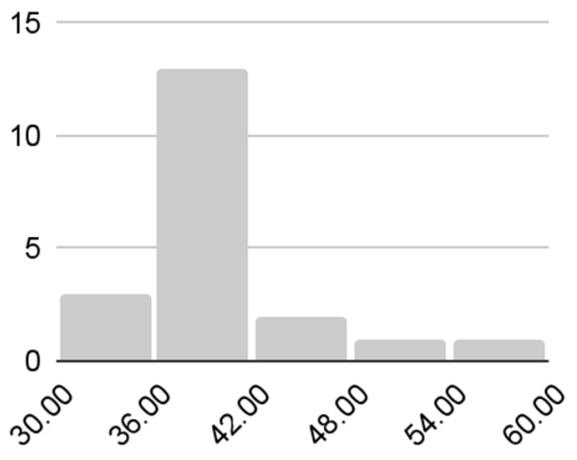

Figure 4: histograms of the data collected from 20 volunteers. From top left to bottom right: a) height of the participants in feet $(1 \mathrm{ft}=30.48 \mathrm{~cm})$ b) bust, c) waist, and d) hips measurements in inches $(1 \mathrm{in}=2.54 \mathrm{~cm})$.

\section{Results and Discussion}

Images and height were used as input data to the algorithm, while measurements provided by volunteers for bust, waist, and hips were used for evaluating the algorithm's performance. We selected bust, hips, and waist measurements as these are the most commonly used in traditional sizing systems. The average difference between Al-generated measurements and tape measurements was less than $3 \%$ and less than $1 \mathrm{in}$. A summary of the results is shown in Table 1. 
Table 1: summary of the differences between Al-generated and tape-measured data for the 20 volunteers included in this study.

\begin{tabular}{|c|c|c|c|}
\hline & Bust & Waist & Hips \\
\hline Median Difference (in) & 1.00 & 0.70 & 1.00 \\
\hline Average Difference (in) & 0.91 & 0.99 & 1.05 \\
\hline Average Difference (\%) & $2.54 \%$ & $3.28 \%$ & $2.69 \%$ \\
\hline Max Difference (in) & 2.60 & 2.40 & 2.29 \\
\hline Min Difference (in) & 0.00 & 0.10 & 0.10 \\
\hline
\end{tabular}

It is worth mentioning that the maximum differences occurred when the user was wearing clothing that had creases, when the lighting was not well diffused (creating shadows around the main image), when the background was extremely cluttered, and that we excluded low-quality images from the study.

The tests performed in this study have shown the importance of good quality images being uploaded by the users. Current mobile devices, generally, have cameras producing images of 3000x4000 pixels, which is a good resolution for this type of application.

On the user experience front, this means the risk of lower image quality is mainly linked to external factors, such as clothes worn, type of lighting, body pose, and position of the phone. A good user interface for this type of application will ensure that customers are given enough information to enable them to take quality photos. User experience is paramount when introducing novel digital technologies, as highlighted in previous research studies [29].

From the designers' point of view, the ability to capture measurements that are within one inch from the customer's body shape enables a more efficient process going from purchase to finished product. Compared to traditional sizes, which can be misleading and result in up to 3 inches difference to the actual measurements [3], this method can be a much better enabler of remote systems for clothing production.

\section{Conclusions}

At The PS Collective, we are creating an inclusive marketplace that wants to highlight women's individuality and style by connecting them with designers who manufacture bespoke and small batches. To achieve this, we are using "PS for you", our inclusive computer vision and Al technology that identifies body measurements designers can use to create custom pieces. This solution is built as an API that can be easily integrated into other platforms, making it highly customizable and available on the cloud at a reduced cost.

By addressing size inconsistency and reliability with online marketplaces, we aim to promote inclusive design and sustainability: the introduction of innovative sizing methods that are adaptable to each individual will reduce the issue of returns, consequently reducing waste.

In the future, we not only want to further improve the accuracy of our models, we'd also like to partner with 3D design software to further automate the process for the designer. This will limit room for human error in manipulating patterns for different body measurements. We will then also be able to use those digital renderings to create a truly immersive and personalized experience for the customer.

\section{Acknowledgments}

This research received no external funding. We'd like to thank the AWS Activate program for providing credits for our cloud development and for consultations through Ubertas Consulting. We'd also like to thank our mentors for feedback on the product, cloud engineer Anna Chiara Faralli PhD for her code review, and study participants for sharing their data with our R\&D team. 


\section{References}

[1] "Size does matter: More than one in three (38\%) Brits struggle to find clothes that fit them well | Mintel.com." https://www.mintel.com/press-centre/retail-press-centre/size-does-matter-more-thanone-in-three-38-brits-struggle-to-find-clothes-that-fit-them-well (accessed Jul. 29, 2021).

[2] Deloitte, "Apparel 2025: What new business models will emerge?," 2020. https://www.deloittedigital.com/content/dam/deloittedigital/us/documents/blog/blog-20200610apparel-trends.pdf

[3] "Not Made to Measure: How to Find Your True Fit in a World of Inconsistent Clothes Sizing | thrEDIT." https://www.thredup.com/bg/p/vanity-sizing?tswc_redir=true (accessed Jul. 29, 2021).

[4] PatrickFarley, "What is Computer Vision? - Azure Cognitive Services." https://docs.microsoft.com/en-us/azure/cognitive-services/computer-vision/overview (accessed Aug. 16, 2021).

[5] "Application of Deep Learning for Object Detection," Procedia Comput. Sci., vol. 132, pp. 17061717, Jan. 2018, doi: 10.1016/j.procs.2018.05.144.

[6] R. Szeliski, Computer Vision: Algorithms and Applications, vol. 1. London: Springer-Verlag, 2011.

[7] M. A. Majdi, B. Sena Bayu Dewantara, and M. M. Bachtíar, "Product Stock Management Using Computer Vision," in 2020 International Electronics Symposium (IES), Sep. 2020, pp. 424-429. doi: 10.1109/IES50839.2020.9231673.

[8] G. Agnihotram et al., "Combination of Advanced Robotics and Computer Vision for Shelf Analytics in a Retail Store," in 2017 International Conference on Information Technology (ICIT), Dec. 2017, pp. 119-124. doi: 10.1109/ICIT.2017.13.

[9] R. A. A. Helmi, A. T. L. Lee, M. G. Md Johar, A. Jamal, and L. F. Sim, "Quantum Checkout: An Improved Smart Cashier-less Store Checkout Counter System with Object Recognition," in 2021 IEEE 11th IEEE Symposium on Computer Applications Industrial Electronics (ISCAIE), Apr. 2021, pp. 151-156. doi: 10.1109/ISCAIE51753.2021.9431839.

[10] W.-H. Cheng, S. Song, C.-Y. Chen, S. C. Hidayati, and J. Liu, "Fashion Meets Computer Vision: A Survey," ArXiv200313988 Cs, Jan. 2021, Accessed: Aug. 02, 2021. [Online]. Available: http://arxiv.org/abs/2003.13988

[11] Julien Ducret, Cédric Claire, Alexis Charlot, Xavier Ameziane, Samuel Cruz-Lara, "Morpheus: a platform for the representation, manipulation and secure access of standardized morphological data for the digital age textile industry," presented at the 11th 3DBody.Tech Conference \& Expo, Lugano, Switzerland, Nov. 2020.

[12] National Institute of Standards and Technology, "Body dimensions for apparel," National Institute of Standards and Technology, Gaithersburg, MD, NIST IR 5411, 1994. doi: 10.6028/NIST.IR.5411.

[13] J. Wells, T. Cole, D. Bruner, and P. Treleaven, "Body shape in American and British adults: Between-country and inter-ethnic comparisons," Int. J. Obes. 2005, vol. 32, pp. 152-9, Feb. 2008, doi: 10.1038/sj.ijo.0803685.

[14] "Clothing Sizes: How Vanity Sizing Made Shopping Impossible." https://time.com/how-to-fixvanity-sizing/ (accessed Aug. 02, 2021).

[15] B. Aboutaleb, "Why Is Inclusive Sizing So Hard?," Racked, Nov. 29, 2016. https://www.racked.com/2016/11/29/13706526/why-is-inclusive-sizing-so-hard (accessed Aug. 16, 2021).

[16] "(PDF) PR Hapss its not in the jeans | Karen Pine - Academia.edu." https://www.academia.edu/2987701/PR_Hapss_its_not_in_the_jeans (accessed Aug. 02, 2021).

[17] S. Grogan, S. Gill, K. Brownbridge, S. Kilgariff, and A. Whalley, "Dress fit and body image: A thematic analysis of women's accounts during and after trying on dresses," Body Image, vol. 10, no. 3, pp. 380-388, Jun. 2013, doi: 10.1016/j.bodyim.2013.03.003.

[18] S. L. Turner, H. Hamilton, M. Jacobs, L. M. Angood, and D. H. Dwyer, "The influence of fashion magazines on the body image satisfaction of college women: An exploratory analysis," Adolescence, vol. 32, no. 127, pp. 603-14, Fall 1997. 
[19] S. Grogan et al., "Body Mass Index and Body Satisfaction: Does Availability of Well-fitting Clothes Matter?," Cloth. Text. Res. J., Feb. 2020, Accessed: Aug. 25, 2021. [Online]. Available: https://www.research.manchester.ac.uk/portal/en/publications/body-mass-index-and-bodysatisfaction-does-availability-of-wellfitting-clothes-matter(5401f62d-f7a0-4665-9ea8265240bafb55).html

[20] E. Saeidi and L. Romeo, "Women's Reaction to Whole 3D Body Scanning and its Influence on Body Satisfaction," Int. Text. Appar. Assoc. Annu. Conf. Proc., vol. 74, no. 1, Art. no. 1, Jan. 2017, Accessed: Aug. 25, 2021. [Online]. Available: https://www.iastatedigitalpress.com/itaa/article/id/1624/

[21] M. PORTER, "How Different Countries Wear Their Suits." https://www.mrporter.com/enus/journal/fashion/how-different-countries-wear-their-suits-873920 (accessed Aug. 17, 2021).

[22] A. Gandhi, C. Magar, and R. Roberts, "How technology can drive the next wave of mass customization," no. 32, p. 8, 2013.

[23] S. Idrees, G. Vignali, and S. Gill, 3D Body Scanning with Mobile Application: An Introduction to Globalise Mass-Customisation with Pakistani Fashion E-Commerce Unstitched Apparel Industry. 2020. doi: $10.15221 / 20.12$.

[24] "Why Size Recommendation Won't Fix Fashion Ecommerce | Linkedln." https://www.linkedin.com/pulse/why-size-recommendation-wont-fix-fashion-ecommerce-bobby\%C3\%B6stberg/ (accessed Aug. 25, 2021).

[25] A. Vecchi, F. Peng, and M. Al-Sayegh, "Size Recommendations in Online Fashion Retail," in Advanced Fashion Technology and Operations Management, 2017. doi: 10.4018/978-1-52251865-5.ch015.

[26] "Deploying machine learning models with serverless templates | AWS Compute Blog." https://aws.amazon.com/blogs/compute/deploying-machine-learning-models-with-serverlesstemplates/ (accessed Aug. 05, 2021).

[27] "Prolific | Online participant recruitment for surveys and market research." https://prolific.co/ (accessed Aug. 25, 2021).

[28] "FastStats - Body Measurements." https://www.cdc.gov/nchs/fastats/body-measurements.htm (accessed Aug. 09, 2021).

[29] S. Miell, S. Gill, and D. Vazquez, "Enabling the digital fashion consumer through fit and sizing technology," J. Glob. Fash. Mark., vol. 9, no. 1, pp. 9-23, Jan. 2018, doi: 10.1080/20932685.2017.1399083. 